\title{
Proteomic Analysis of Loricrin Knockout Mouse Epidermis
}

Robert H. Rice, ${ }^{*,}{ }^{\dagger}$ Blythe P. Durbin-Johnson, ${ }^{\ddagger}$ Yosuke Ishitsuka, ${ }^{\S}$ Michelle Salemi, ${ }^{\|}$Brett S. Phinney," David M. Rocke, ${ }^{\ddagger}$ Dennis R. Roop ${ }^{\S}$

$\dagger$ Department of Environmental Toxicology, University of California, Davis, CA 95616-8588

$\ddagger$ Department of Applied Biosciences, University of California, Davis, CA 95616

$\S$ Department of Dermatology, Charles C. Gates Center for Regenerative Medicine and Stem Cell Biology, University of Colorado Anschutz Medical Campus, Aurora, CO

\|Proteomics Core Facility, University of California, Davis, CA 95616-8588

Table of Contents

Figure S1. Multidimensional scaling plots for the envelope and total cross-linked samples.

Figure S2. Proteins with reduced incorporation into envelopes in Lor KO samples harvested 4 days after birth.

Figure S3. Proteins with increased incorporation into envelopes in Lor KO samples harvested 4 days after birth. 
Figure S1. Multidimensional scaling plots for the envelope and total cross-linked samples. KO1, KO4= samples from Lor knockout mice 1 or 4 days after birth, respectively. WT1, WT4 = samples from wild type mice 1 or 4 days after birth, respectively.
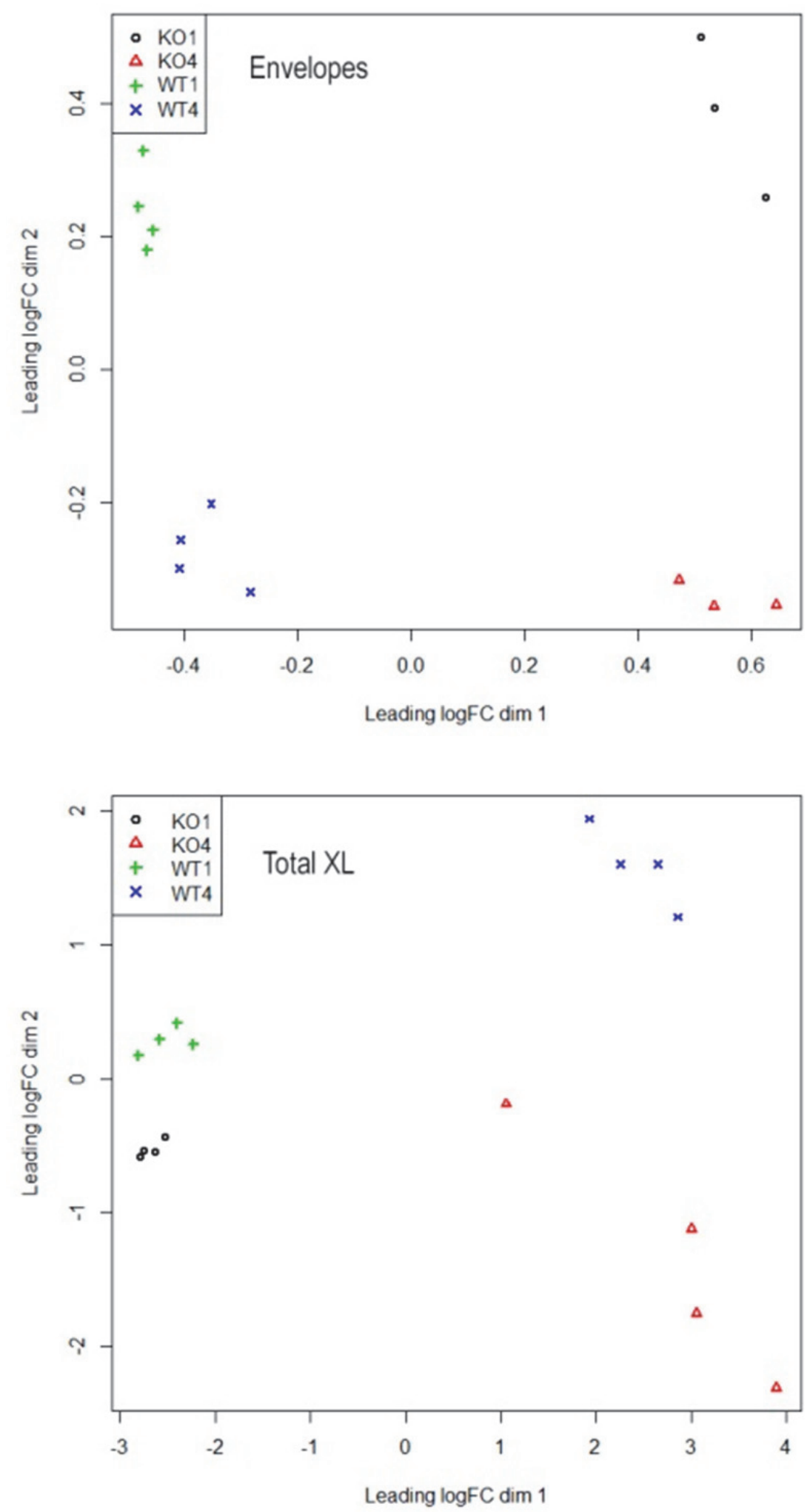
Figure S2. Proteins with reduced incorporation into envelopes in Lor KO samples harvested 4 days after birth.

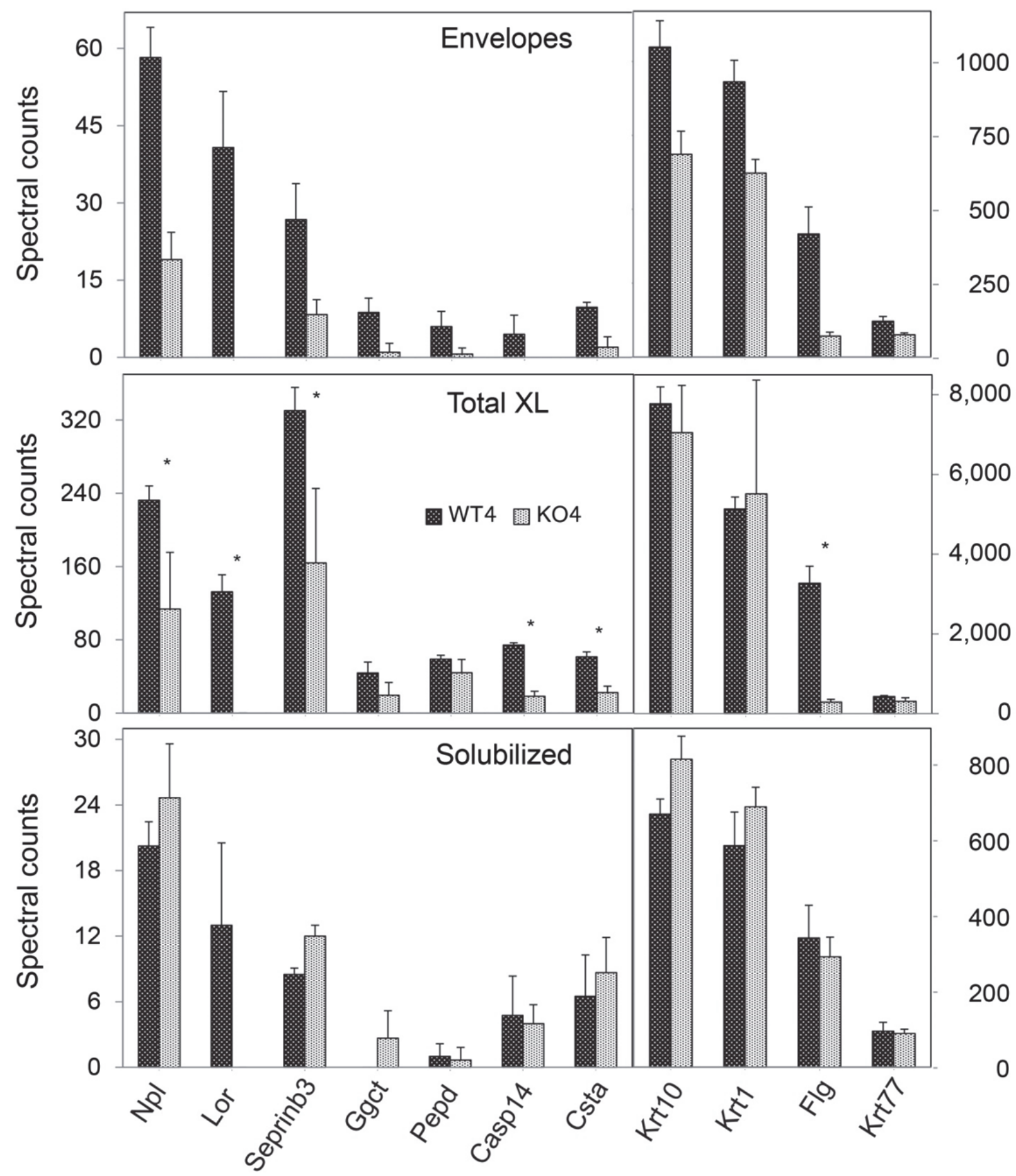


Figure S3. Proteins with increased incorporation into envelopes in Lor KO samples harvested 4 days after birth.
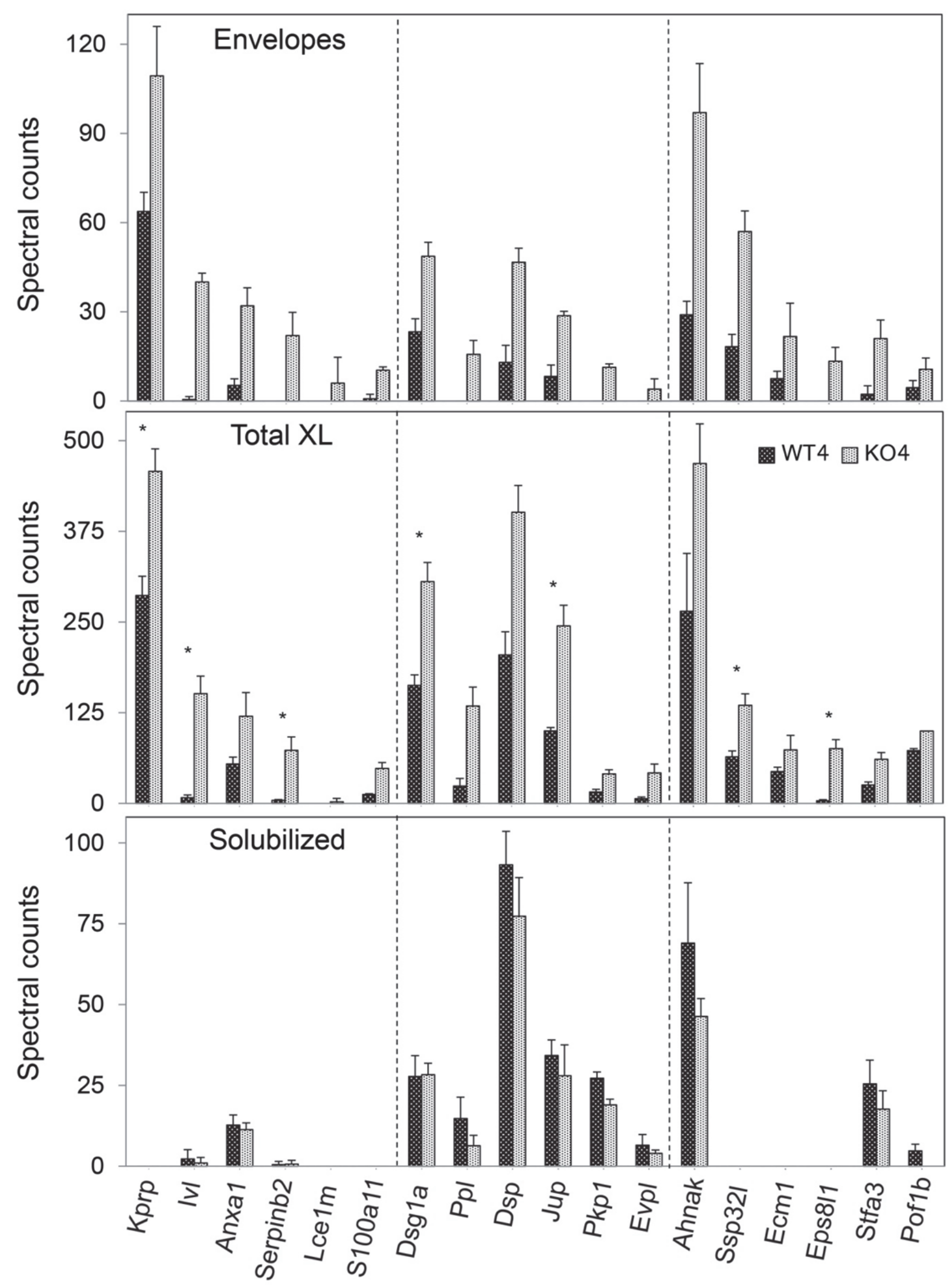